\title{
Esophageal Retention with Retrograde Flow Below Pharyngoesophageal Segment Following Swallowing
}

National Cancer Institute

\section{Source}

National Cancer Institute. Esophageal Retention with Retrograde Flow Below

Pharyngoesophageal Segment Following Swallowing. NCI Thesaurus. Code C127346.

A finding of esophageal retention with retrog rade flow below the pharyngoesophageal segment. 